\title{
Differential Involvement of ASIC1a in the Basolateral Amygdala in Fear Memory and Unconditioned Fear Responses
}

\author{
Stéphanie Grégoire and Julien Matricon \\ Laboratoire de Pharmacologie Fondamentale et Clinique de la Douleur, Université d'Auvergne, Institut National de la Santé et de la Recherche Médicale, \\ Unité Mixte de Recherche 766, 63000 Clermont-Ferrand, France \\ Review of Coryell et al. (http://www.jneurosci.org/cgi/content/full/28/51/13738)
}

Emotion is critical to provide an adequate response to the environment. Hyperemotionality induces strongly invalidating psychiatric diseases such as posttraumatic stress disorders or phobias. Investigating how the brain controls and regulates fear expression is crucial to better understand these troubles.

Fear conditioning is the main paradigm used in clinical and preclinical research to investigate the mechanisms underlying fear learning and fear memory. Classical or pavlovian fear conditioning results from the association of a conditioned stimulus (CS), initially neutral, and a noxious unconditioned stimulus (US), usually an aversive footshock (fear training). This association drives the development of defensive conditioned responses following a reexposure to the CS (fear memory).

Surgical, pharmacological, and electrophysiological studies have highlighted neuronal pathways underlying associative learning, especially the limbic system (hippocampus, amygdala) and related cerebral structures (prefrontal, perirhinal,

Received Feb. 13, 2009; revised April 2, 2009; accepted April 3, 2009.

Correspondence should be addressed to Stéphanie Grégoire, Laboratoire de Pharmacologie Fondamentale et Clinique de la Douleur, Université d'Auvergne, Institut National de la Santé et de la Recherche Médicale, Unité Mixte de Recherche 766, 63000 Clermont-Ferrand, France. E-mail: stephanie.gregoire@u-clermont1.fr.

DOI:10.1523/JNEUROSCI.0752-09.2009

Copyright $\odot 2009$ Society for Neuroscience $\quad$ 0270-6474/09/296053-02\$15.00/0 and entorhinal cortices). Among these sites, the amygdala is notably involved in encoding and storing the "CS-US" association. The basolateral amygdala complex (BLA), composed of the lateral, the basolateral, and the basomedial nuclei, is of particular interest since BLA inactivation using neurotoxic lesions impairs the occurrence of cue or contextual conditioned fear behavior (Maren, 2001). CS-US association in the BLA relies on NMDA receptor-dependent long-term potentiation (LTP) mechanisms and the activation of the PKA-CaMKII downstream signaling cascade (Kim and Jung, 2006). However other mechanisms might be involved. For example, intra-amygdala infusion of the $\mathrm{GABA}_{\mathrm{A}}$ receptor agonist muscimol in rats or disruption of functional metabotropic glutamate receptors, mGluR1 and mGluR3, in mice impair pavlovian fear conditioning.

ASICla, an acid-sensitive ion channel widely expressed in the central and peripheral nervous system, has a key function in mechanosensation and nociception. Interestingly, a role in fear learning and memory is also emerging. Recently, ASICla has been shown to be essential to innate fear but also to fear conditioning behaviors (Coryell et al., 2007). Considering the high ASIC1a expression level in the BLA, Coryell et al. (2008) hypothesized that (1) ASICla could be involved in neuronal mechanisms associated with fear learning and that, consequently, (2) disrupted fear memory in mice lacking functional ASIC1a (ASICla-/-) could be rescued by a specific viral administration of the ASICla gene in the BLA (Coryell et al., 2008).

To determine the role of ASICla in fear memory, the authors applied context fear conditioning to mice deficient for the ASICla gene. Context fear conditioning consisted in the association of a conditioning chamber with five footshock series (1 s, $0.75 \mathrm{~mA}$ ). The total time during which an animal exhibited freezing behavior was used as a measure of context fear conditioning.

To assess whether ASICla in BLA is indeed involved in fear training, the expression level of the protein cFos was evaluated following context fear conditioning. ASICla $-/-$ mice showed decreased c-fos expression compared with wild-type (WT) mice, suggesting that the deficit in ASIC1a decreased neuronal activity in BLA [Coryell et al. (2008), their Fig. 1].

To test more directly whether fear conditioning requires ASIC1a activation in the BLA, the authors investigated the impact of rescuing ASIC1a expression specifically in the BLA of ASICla-/- mice. Adeno-associated virus (AAV)-mediated gene transfer was used to restore ASICla expression bilaterally in the BLA. WT and ASICla-/- mice treated with AAVASICla virus or control, a vector contain- 
ing the green fluorescent protein (AAVGFP), were trained and tested to the context-footshock conditioning.

As previously shown (Wemmie et al., 2003), ASIC1a $-/-$ mice froze less often than WT mice during the training phase. Moreover, rescuing ASIC1a expression in the BLA was not sufficient to restore the WT level of freezing [Coryell et al. (2008), their Fig. $2 E$ ]. This suggests that the role of ASIC1a in fear training involves cerebral structures other than BLA.

The day after training, mice were returned to the conditioning chamber to test context-evoked freezing. Untreated ASIC1a-/- mice froze less than WT mice during testing. Surprisingly, the conditioned freezing in AAV-ASICla-treated mutant mice was the same as that of WT [Coryell et al. (2008), their Fig. 2 F], indicating that ASIC1a expressed in the BLA plays a crucial role in context-dependent conditioned fear responses. This result raises the question of how AAV-ASIClatreated mice could display an appropriate fear-conditioned response while they still present fear training deficits? This would imply that fear memory and fear training rely on different ASICla-dependent memory mechanisms.

Results of the ASIC1a rescue experiments suggest that ASICla activity in the BLA plays a role in fear conditioning, but raises the perplexing question of why the conditioned response (CR) was larger than the unconditioned response (UR). Given that ASICla has been shown to be involved in nociception (Mazzuca et al., 2007) and to contribute to pain-elicited currents in the spinal cord $(\mathrm{Wu}$ et al., 2004), ASICla-/- mice are likely to present nociceptive sensory deficits. This could involve a weakened perception of pain which could consequently impair the CS-US pairing.

It thus appears essential to further assess ASIC1a-/- mice nociceptive sensitivity using acute pain models, for example the hot-plate test. Indeed, a somatic painful stimulation such as intraplantar injection of formalin induces increased c-fos mRNA expression in the BLA (Nakagawa et al., 2003). Consequently, the decreased c-fos expression observed in ASIC1a-/- mice BLA following footshock-context conditioning could arise from a reduced response to pain. In contrast, a similar pain threshold of ASICla-/- and WT mice would suggest that freezing deficits during the training phase result from modified emotional responses to pain or modified ASICladependent fear training mechanisms. Since BLA treatment with ASIC1a-AAV did not rescue freezing deficits, these mechanisms are likely to involve ASIC1a activation in other cerebral regions such as the medial prefrontal or the entorhinal and perirhinal cortices which are known to play a key role in context fear memory. Viral transduction of ASIC1a-AAV in these structures before contextual fear learning could provide answers to this issue.

In another experience, Coryell et al. (2008) attempted to determine whether all unconditioned responses were impaired in ASICla-/- mice. The nonpainful US (predator odor) produced the same deficit in freezing as the painful one [Coryell et al. (2008), their Fig. 2G]. This result supports the hypothesis that the strength of the CS-RS is not directly tied to the strength of the US-UR. Nevertheless, to avoid the confusing influence of pain perception on memory processes, the authors could have used a nonpainful aversive conditioned stimulus (e.g., predator odor). However, any US that evokes a fear response would also produce anxiety. A recent study demonstrated that ASICla antagonists have anxiolytic effects (Dwyer et al., 2009). Consequently, less freezing during training in ASICla-/- mice could be interpreted as a loss of ASICla anxiolytic properties. Anxiety levels in mice could be evaluated using an elevated-zero maze, which is relevant due to the prominent role of amygdala in emotional behavior.

The mismatch between ASICla-/mice freezing during testing and the expression of long-lasting fear memory during training remains to be elucidated. As the test was performed $24 \mathrm{~h}$ following the training, plasticity mechanisms such as consolidation could have occurred during this period and contributed to the formation of a stable memory. To test this hypothesis, the time interval between training and testing could be reduced (to 30 min) or one could consider using ASIC1a antagonists following the training phase. Thus, preventing the consolidation would blunt the freezing ASIC1a-AAV rescue observed during testing.

In summary, Coryell et al. (2008) provide evidences that ASIC1a is not solely involved in context-dependent fear behavior and that the site of ASICla action is likely to be the BLA. Strikingly, these results report that expressing ASIC1a in the BLA of ASIC1a-/- mice rescues fear memory but not the freezing deficit observed during training. This study raises several questions for future investigations. A better understanding of how "CS-US" association is established and determining the contribution of different brain structures like BLA to fear memory would help the development of new behavioral or pharmacological strategies to treat pathological fears.

\section{References}

Coryell MW, Ziemann AE, Westmoreland PJ, Haenfler JM, Kurjakovic Z, Zha XM, Price M, Schnizler MK, Wemmie JA (2007) Targeting ASIC1a reduces innate fear and alters neuronal activity in the fear circuit. Biol Psychiatry 62:1140-1148.

Coryell MW, Wunsch AM, Haenfler JM, Allen JE, McBride JL, Davidson BL, Wemmie JA (2008) Restoring acid-sensing ion channel-1a in the amygdala of knock-out mice rescues fear memory but not unconditioned fear responses. J Neurosci 28:13738-13741.

Dwyer JM, Rizzo SJ, Neal SJ, Lin Q, Jow F, Arias RL, Rosenzweig-Lipson S, Dunlop J, Beyer CE (2009) Acid sensing ion channel (ASIC) inhibitors exhibit anxiolytic-like activity in preclinical pharmacological models. Psychopharmacology (Berl) 203:41-52.

Kim JJ, Jung MW (2006) Neural circuits and mechanisms involved in pavlovian fear conditioning: a critical review. Neurosci Biobehav Rev 30:188-202.

Maren S (2001) Neurobiology of pavlovian fear conditioning. Annu Rev Neurosci 24:897-931.

Mazzuca M, Heurteaux C, Alloui A, Diochot S, Baron A, Voilley N, Blondeau N, Escoubas P, Gélot A, Cupo A, Zimmer A, Zimmer AM, Eschalier A, Lazdunski M (2007) A tarantula peptide against pain via ASICla channels and opioid mechanisms. Nat Neurosci 10:943-945.

Nakagawa T, Katsuya A, Tanimoto S, Yamamoto J, Yamauchi Y, Minami M, Satoh M (2003) Differential patterns of c-fos mRNA expression in the amygdaloid nuclei induced by chemical somatic and visceral noxious stimuli in rats. Neurosci Lett 344:197-200.

Wemmie JA, Askwith CC, Lamani E, Cassell MD, Freeman JH Jr, Welsh MJ (2003) Acidsensing ion channel 1 is localized in brain regions with high synaptic density and contributes to fear conditioning. J Neurosci 23: 5496-5502.

Wu LJ, Duan B, Mei YD, Gao J, Chen JG, Zhuo M, Xu L, Wu M, Xu TL (2004) Characterization of acid-sensing ion channels in dorsal horn neurons of rat spinal cord. J Biol Chem 279: 43716-43724. 